\title{
Supporting Sandtray Therapy on an Interactive Tabletop
}

\author{
Mark Hancock ${ }^{1}$, Thomas ten Cate ${ }^{1,2}$, Sheelagh Carpendale ${ }^{1}$, Tobias Isenberg ${ }^{2}$ \\ ${ }^{1}$ University of Calgary, Canada \\ Department of Computer Science \\ $\{$ msh,sheelagh\}@cpsc.ucalgary.ca

\begin{abstract}
${ }^{2}$ University of Groningen, The Netherlands
Institute of Mathematics \& Computer Science
\end{abstract} \\ t.ten.cate.1@student.rug.nl,isenberg@cs.rug.nl
}

\begin{abstract}
We present the iterative design of a virtual sandtray application for a tabletop display. The purpose of our prototype is to support sandtray therapy, a form of art therapy typically used for younger clients. A significant aspect of this therapy is the insight gained by the therapist as they observe the client interact with the figurines they use to create a scene in the sandtray. In this manner, the therapist can gain increased understanding of the client's psyche. We worked with three sandtray therapists throughout the evolution of our prototype. We describe the details of the three phases of this design process: initial face-to-face meetings, iterative design and development via distance collaboration, and a final face-to-face feedback session. This process revealed that our prototype was sufficient for therapists to gain insight about a person's psyche through their interactions with the virtual sandtray.
\end{abstract}

\section{ACM Classification Keywords}

H.5.3 Information interfaces and presentation: Group and Organization Interfaces- $C S C W$.

\section{General Terms \\ Design, Human Factors}

\section{INTRODUCTION}

Social workers and therapists are developing new ways of reaching and working with children who are troubled or have experienced traumatic events and difficult life situations. One such method is sandtray therapy $[3,7,16]$ - a type of play or art therapy [5] in which the use of figurines in a tray of sand is a vehicle for establishing interaction and rapport between the therapist and the child (Figure 1). Children placing and moving the figurines in the sandtray provides a venue by which therapists can observe the manner in which the child thinks about their experiences and feelings. In response to an idea from a practicing sandtray therapist, we developed a virtual sandtray. In this paper we present its iterative evolution in collaboration with three therapists who make use of sandtray in their therapeutic practices.

Permission to make digital or hard copies of all or part of this work for personal or classroom use is granted without fee provided that copies are not made or distributed for profit or commercial advantage and that copies bear this notice and the full citation on the first page. To copy otherwise, or republish, to post on servers or to redistribute to lists, requires prior specific permission and/or a fee.

CHI 2010, April 1015, 2010, Atlanta, Georgia, USA.

Copyright 2010 ACM 978-1-60558-929-9/10/04...\$10.00.



Figure 1: A sandtray typically used for sandtray therapy. Sandtray therapists typically observe clients creating a scene or "playing" in the sand to gain insight into their psyche (courtesy of Kristina Walter [29]).

There were many reasons for the request that triggered this research. Sandtray therapy, often considered a type of art therapy [5] because the therapy session involves the clients creating a scene out of available supplies, has particular characteristics which make it well suited to an interactive tabletop. These include factors of age stereotyping, the characteristics of the sand itself, and the types of interaction that are therapeutically beneficial. In terms of age stereotyping, the associations of sandtrays (or sandboxes) are with activities we did as young children. While this works well in establishing rapport with young children, it can pose problems with young teenagers and pre-teens to whom activities in a sandbox may seem just too uncool. This age group (10 to about 13 or 14) is a particularly difficult age group for therapists to reach and is also a particularly sensitive age group since so much is changing in their lives at these ages. One of the therapists we are working with suggested that the "wow' factor of a digital table might prove a great bridging factor. Also, some children, perhaps due to their own response to traumas, dislike the feeling of sand and refuse to play with it and may find a digital sandbox more to their liking.

Perhaps most important is the potential for interaction; sandtrays offer special interaction advantages. For example, other forms of media used in art therapy [5], such as pencils, paints, and clay, result in the client creating a 'snapshot' (e.g., pencil drawings, paintings, sculptures) as an end result. In contrast, the temporary nature of the sand invites play and, therefore, the creation of a narrative. The process of creating a 'scene' containing several objects, in which the narrative can unfold, can be particularly informative to the therapist, who can often infer self-representation in one object and, from there, the relationships to other objects in 
the scene. Thus, the client's interaction with the objects is of particular importance to the therapist, and the possibilities of multi-touch interaction make this application particularly suitable to adoption with tabletop display technology. The direct nature of touch input to tabletop displays affords observation of these interactions, and the fact that the display is digital makes the scene being created similarly temporary.

While this is an interesting application for tabletops, there are also particularly interesting and challenging research issues. The therapists we worked with explained how in the physical world they have become sensitive over time to understanding the possible implications of the active (manipulation of physical objects) part of the sandtray work. We were particularly interested in whether this professional skill would hold for virtual 3D interaction. In particular, is the virtual medium rich enough for a scene to be constructed that the therapists can understand, or are viewing issues, such as the need to project onto a $2 \mathrm{D}$ screen, too limiting? Can a virtual object take on a variety of meanings so as to enable the telling of a story to the therapists, or will they be interpreted as mere data or information? Are the interactions on a virtual table rich enough to convey meaning to the therapist about a client's psyche, or is the disconnect between a person's actions and the surface's reaction too great? Our more general goal was to discover whether the therapists felt that they could effectively perform therapy with this digital sandtray or some future design iteration.

We present the cooperative design of a virtual sandtray through three phases of design: initial face-to-face meetings, iterative remote collaborative design, and a face-to-face feedback session. We end with an in-depth discussion of the results of this collaboration and a description of how to make use of our findings beyond the digital sandtray.

\section{RELATED WORK}

In addition to the field of traditional sandtray therapy [3, 5, $7,16]$, we review relevant research in the following areas: technologies that support storytelling or therapy, and tabletop interaction with both $2 \mathrm{D}$ and $3 \mathrm{D}$ virtual objects.

\section{Storytelling and Therapeutic Tabletop Applications}

Our specific application domain, virtual sandtray therapy, is related to a number of approaches where modern touch and tangible technology is used to support work with children for storytelling or therapeutic purposes. Early examples include the use of robotic stuffed animals [2] to help young cardiac patients cope with their situation by encouraging them to talk about it, comparable to virtual sandtray therapy. Later work employed an interactive physical play mat (StoryMat) to record children's storytelling activities [6,24]. Li et al. [20] developed a tangible tabletop game to support the therapy of children with cerebral palsy who need to train specific motor skills. The game combined tangible elements with a tabletop surface that was illuminated with coloured LEDs from below and was found to encourage children to train the desired therapeutic movements. Morris et al. [22] describe an interface designed for children with Asperger's syndrome. Here, the fact that tabletop displays afford collaboration is used in a game form to allow four children simultaneously to train social skills and collaboration. Similar to our own motivation, the authors name the children's affinity to technology as one of the criteria that makes tabletop technology well suited for such therapeutic applications.

Although not used to tell a story, Piper and Hollan [23] describe the design of an interactive table used to facilitate communication between a doctor and a deaf patient. This design process has many similarities to our own and shows the benefit of tabletop technology for the deaf community. As would be expected, our work reinforces the idea that working closely with the people who professionally understand the application area can lead to successful tabletop applications.

The focus of our research differs from these storytelling and therapeutic tabletop applications. We focus on the therapists and their ability to interpret the actions being carried out on the tabletop display itself. We leverage existing literature that suggests the usability of our interaction techniques and ask whether these techniques are rich enough to be interpreted by the observing therapist.

\section{D Tabletop Display Interaction}

Kruger et al. [17] showed that people use the orientation of artifacts on a table to communicate with one another. This form of silent communication is likely highly relevant to the therapist-client communication process in sandtray therapy, as the orientation of objects in the sandbox can be a key element of interpreting a client's thoughts. Their rotate n' translate (RNT) technique [18] uses only one point of contact to enable this rich communication. Techniques such as TNT [21] have extended this technique by mapping the rotation of a hand or stylus to the orientation of the virtual object. As modern touch technologies (e.g., $[8,11])$ provide more simultaneous touches, these additional contact points can also be used to specify object position, orientation, and scale. For example, a technique now commonly used in multi-touch consumer appliances employs two points of contact to specify position, orientation, and size of objects [13]. Depending on the hardware that recognizes the input and on the task to be performed, however, the suitability of the different interaction techniques may vary [13].

In addition to applying simple transformations to objects, it is often necessary to invoke other actions. While techniques known from traditional interfaces such as menus, tool palettes, and buttons can be used for this purpose, touch interfaces often also employ gestures. For example, $\mathrm{Wu}$ and Balakrishnan [32] demonstrated how to control a room planning application using a variety of gestures and hand postures. Other approaches also combine posture and gesture interaction with speech input [28]. Even with relatively simple size-based recognition hardware it is possible to use a set of hand postures to parameterize or control actions in an interface [10]. Input techniques that provide more information on the shape of a touch, however, can be used to define postures inspired by physical interaction and to infer forces to be used in the interaction [4]. An approximated touching force can also be used to control the layering of $2 \mathrm{D}$ 
objects [2], which is related to the concept of shallow-depth 3D interaction [12] on which our work is largely based.

We leverage some of this work by providing interaction techniques that allow the therapist to interpret the actions of the client. In our system, we opted for interaction techniques that explicitly convey the consequence of the action, as opposed to abstract gestures that must be learned. We also chose to use physically familiar feedback from the system as opposed to (for example) drastic global changes caused by subtle actions, such as a button press.

\section{D Tabletop Display Interaction}

While interaction techniques that rely on input captured in 3D space have been investigated in detail in 3D stereoscopic environments such as the Responsive Workbench [19], we concentrate on 2D input on the surface of a horizontal display to interact with a 3D scene. In particular, we use sticky fingers and opposable thumbs [14], a technique based largely on shallow-depth 3D techniques [12], to provide six degrees of freedom (DOF) control of 3D objects. This shallow-depth technique has been previously evaluated, demonstrating that three touches can be both faster and more well-liked than one or two touches. Another metaphor to translate 2D input to interaction with 3D shapes in a horizontal interface, BumpTop, was introduced by Agarawala and Balakrishnan [1]. This interface is based on a physics simulation with 3D shapes, controlled through gestures as well as some menus. A similar interface that also relies on physical simulation was introduced by Wilson et al. [31] who extend the interacting objects such as fingers virtually into a 3D environment in which objects are located. This system was also evaluated and shown to be faster and well-liked by participants.

We leverage these 3D interaction techniques together with the use of a physics engine to provide tabletop interaction that is interpretable by the therapist. While these techniques and their corresponding studies show the usability of 3D interaction on a table, our work shows that these same interaction techniques can also benefit the therapist observing these interactions. By striking a balance between the ability to precisely control the 3D object and having on-screen objects react physically to those precise actions, the actions of the client become rich enough for the therapist to interpret the 'language' of those actions from the client.

\section{METHODOLOGY}

We used a cooperative design process $[9,26]$ with three sandtray therapists, who were involved throughout this research to provide us with expert domain knowledge. There were three phases of this design process: Phase I involved initial face-to-face meetings with one sandtray therapist (T1), Phase II was an iterative distance collaboration (via phone and email) with two therapists (T1 \& T2) throughout the design and implementation of the prototype, and Phase III was a face-to-face discussion with two therapists (T1 \& T3) to provide feedback about the working prototype. The first phase involved two meetings, a pre-planning meeting and a follow-up meeting. At the former meeting, the idea of implementing a digital sandtray had not yet been conceived.
This meeting was a demonstration of existing technology to T1, which triggered a discussion about how a digital tabletop sandtray might provide a solution to some of the comfort problems some clients feel with traditional sandtrays. The second meeting was to plan how we could collaboratively design our prototype at a distance, as the therapists were in a city approximately $630 \mathrm{~km}$ from our research lab. The second phase in our design process involved extensive discussion via phone and email with both $\mathrm{T} 1$ and $\mathrm{T} 2$, who described in detail what they felt were the essential elements of sandtray therapy. We include direct quotes from our email communication with T2 throughout the next section (Phase I \& II: Designing the Virtual Sandtray). We responded to this communication with descriptions of design ideas and questions about what made the elements important. These conversations were highly iterative and led to the design considerations that are described next. During design and development of the prototype, we maintained contact with both T1 and $\mathrm{T} 2$ to iterate and refine the design. When the prototype was finished, T1 and T3 joined us in a day-long workshop to use our prototype first-hand and provide feedback (Phase III: Face-to-Face Feedback Session).

Our in-depth discussions with the therapists revealed that it is common for them to constantly be collecting artifacts to use in therapy sessions from the environment (e.g., sticks, leaves, plastic cups, etc.). For example, one could consider our first meeting with T1 to be an example of her 'collecting' our technology. More generally, their practice frequently involves the evaluation of the suitability of tools and techniques for use in therapy. This skill is learned over time and does not directly involve their clients. We thus focused our research on the therapists; our research asks whether, in the virtual world, therapists can still interpret a person's actions in a meaningful way to perform therapy. We therefore decided to only include therapists (and not clients) in our design process. This decision has the consequence that our findings should be interpreted with a therapist-focused lens. We did, however, include a mock therapy session in our daylong workshop, where one of the designers played the role of a client. Indeed, part of the training for students learning to do sandtray therapy involves participating in a session as a client themselves. The therapists in our study explained that these mock therapy sessions were necessary in order to better understand the experience for the client. Thus, it seemed particularly appropriate for our own understanding to undergo a similar experience. We rely on previous studies $[12,31]$ to validate the usability of the interaction techniques used by the client.

\section{PHASE I \& II: DESIGNING THE VIRTUAL SANDTRAY}

There are two possible avenues to explore for features in a digital sandtray: those already offered by a physical sandtray, perhaps adapted for use on a tabletop, and new options that do not exist in the physical world, but are made possible by the virtual. Our list of design considerations (DC1-DC4) contains some features from traditional sandtrays that the therapists thought were important to maintain and some digital features they thought it would be interesting to explore. In our bottom-up approach we started with nothing, adding 


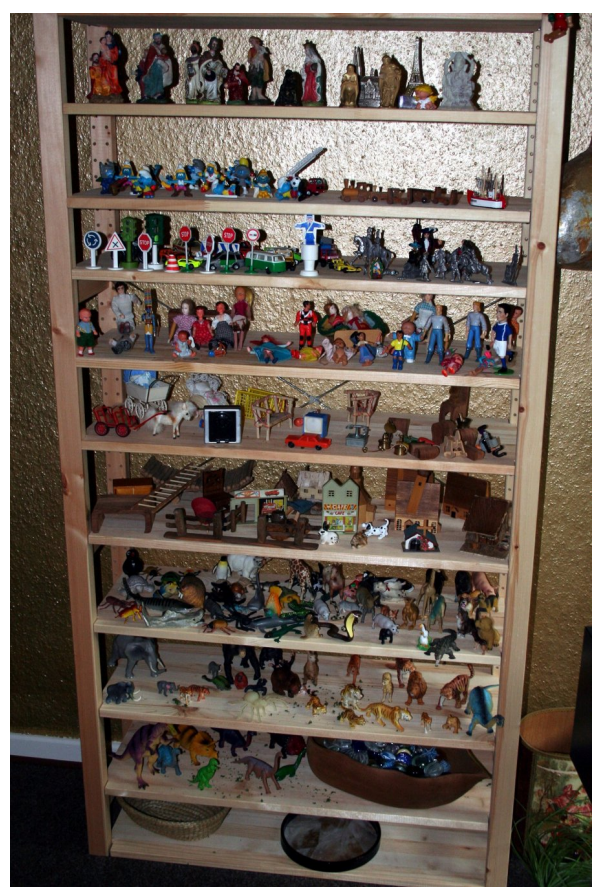

Figure 2: A shelf of figurines used for sandtray therapy (courtesy of Kristina Walter [30]).

features that are deemed valuable for therapy, until a sufficiently rich environment was created. Our communication with sandtray experts was the main guide in determining the most worthwhile features. A secondary concern was the cost of a feature in terms of interface and interaction complexity.

DC1: maintain narrative potential — without characters, there can be no story; thus, the use of figurines to represent characters and objects is essential (Figure 2). It must be possible to add figurines when a new character or object is introduced, to move them around as the story progresses, and to remove them when their part is over. Stacking objects, such as a balanced stack of rocks or an animal on a house, also has significant psychological connotations.

"[T]he temporary and unfixed nature of the sand-tray pieces invites play and therefore the creation of narrative. Most other media result in a 'snapshot' in which the narrative is implied but not played out." -T2

DC2: maintain the sandtray's characteristic as primarily an associative medium — with open media, such as paint and clay, the artist (client) has a sense of the meaning as coming from and being expressive of themselves. With a sandtray, meaning is primarily associated with the objects, and usually only one of the objects is the self representation. Thus, to enable the development of associated meaning that allows the client to express their particular story, a broad range of objects or figurines is desirable. Some therapists group the figurines they offer by category, which makes it easier to find related figurines; others prefer a completely random presentation in which all figurines are mixed, which can trigger more spontaneous associations. Although a digital system could allow both options, we chose to use a random presentation to encourage free association. From a commercial library of 3D models, around 160 figurines of many different categories were selected for use in the virtual sandtray.

"With a sand tray it is rare that a client will ask for a specific object, precisely because inspiration tends to start with associations to the presented repertoire of objects. This puts the client in the position of immediately symbolizing and associating. While the (relatively) fixed nature of the objects limits the expressiveness of the work, the way that they are animated and placed becomes the client's means of articulating their own meanings regarding those objects." $-\mathrm{T} 2$

However, the sand itself can be used as an open medium that can be shaped at will and made into a backdrop for the story. Because directly simulating the behaviour of sand is computationally intensive, we decided to provide a different type of background open media in textured 'paint'. The specific types of paint we included represent different surfaces, such as sand, grass, concrete and water.

Also the temporary nature of the sand was described by the therapists as being important. When a client sees a box of sand, they immediately recognize that whatever they create in that sandbox can be easily erased with a simple swipe of the hand. A digital display is in this way similar to a physical sandbox, because the pixels drawn on the screen are also in some sense temporary. By clearing the screen or turning off the monitor, whatever the client creates can be easily erased. Recording is still possible, much like a sandtray session can (and often is) videotaped.

DC3: exploring simple digital extensions - much of the design discussion with the therapists considered which aspects of digital magic might make useful enhancements. In the real world, it is not possible to instantly duplicate objects. In the digital world, it is trivial. Being able to add multiple copies of the same figurine allows for the creation of forests, herds and families, with little or no cost in terms of interface complexity. The sandtray therapists described this feature as being particularly worthwhile.

Another real-world impossibility is resizing rigid objects (again, digitally trivial). The size of an object has significant psychological connotations: larger objects are perceived as more important, more powerful or more menacing.

“... resizability could be a huge advantage of a virtual play-table. It's an ongoing issue that my toy collection features a range of scales. I have some dinosaurs that are smaller than my cockroaches. Children adapt and play with this, and it sometimes suggests interesting possibilities (like a giant baby who rescues a mom from a tiny car), but scalability would give you the best of all possible worlds... It's such a rich metaphor: the sense that in our psychological/creative world things do not have 'realistic' sizes, they have metaphorical sizes."-T2

DC4: use multi-touch to facilitate interpretation in the therapist-client collaboration - The interactions between the client and the sandtray are the focus of the therapist's 
observations, and thus a key aspect of this collaboration is the awareness by the therapist of these interactions. Furthermore, sandtray therapy is sometimes used for couples or families, who will cooperatively act out a story, and even a single client can ask the therapist to participate. We must also design for such multi-person scenarios.

In the next three sections, we describe in detail how we realize our design considerations in our implementation of the virtual sandtray. We adapt existing tabletop display techniques and technology to provide rich interactions so the therapist can easily observe and infer information about the client's psyche from their interaction. Design principles (DC1-DC4) are indicated for each implementation detail.

\section{Implementation Details}

We implemented our prototype using the SMART Table ${ }^{1}$, which was specifically designed for children. Its small form factor ensures that all corners of the table can be reached by a child, and its sturdy design makes it suitable for use in a practical setting. Moreover, it uses frustrated total internal reflection (FTIR) [11] and can detect up to 40 simultaneous touches, enabling interaction through multiple fingers for multiple people at once. The direct nature of multitouch technology supports awareness by the therapist of the client's interactions (DC4). These factors make the SMART Table an ideal choice of hardware.

“...the principle is that a sand-tray should be just big enough to fill the field of vision. This gives the sense of an immersive world without requiring that the user look around to take the whole thing in... My sandtray is about the size that you suggest $(75$ by $52 \mathrm{~cm}$ ), and that feels about right." $-\mathrm{T} 2$

To enhance both the feeling of realism, and the narrative abilities (DC1), we employ NVIDIA's PhysX physics simulation engine ${ }^{2}$ in a similar way to Wilson et al. [31]. This allows clients to make figurines fall down, roll around, knock each other over, and to toss them around without any extra interaction techniques or development effort.

\section{Figurine Manipulation}

The combination of precise control over the object being moved, together with the physical reaction of the remainder of the scene, provides the client with a platform for rich expression through their narrative (DC1). With this system, the physical movements of the client have a direct correlation with the response by the system, allowing the physical movements to be interpreted by the therapist.

"... most children will depict battles at some point. Different varieties of aggression may be coming out in this..." $-\mathrm{T} 2$ "positioning objects-includes orientation and ability to push into sand." $-\mathrm{T} 2$

${ }^{1}$ SMART Technologies. http://www.smarttech.com/

${ }^{2}$ NVIDIA Corp. http://www.nvidia.com/object/nvidia_physx.html

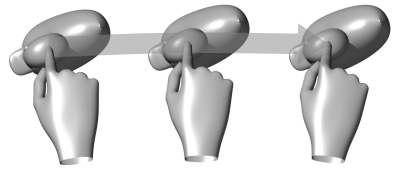

(a)
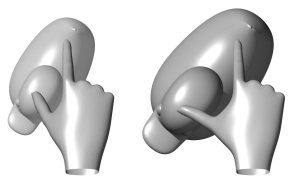

(c)
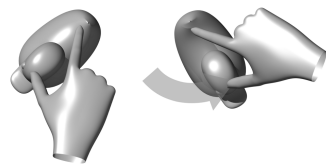

(b)

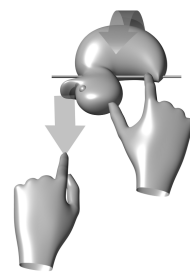

(d)
Figure 3: The first finger (a) moves the figurine in the plane. The second finger rotates (b) about and moves (c) the figurine along the $z$ axis. The third finger (d) rotates the figurine about the $x$ and $y$ axes.

"moving objects-sometimes includes momentum, especially when throwing objects and lifting/dropping them."

"*stacking objects* (small objects placed on top of larger ones, balanced stacks of rocks, animals in trees or on houses etc.)"

$-\mathrm{T} 2$

"children love containment, frequently putting things under or within other things. Another powerful metaphor"

To support this rich narrative, the client should be able to freely move and rotate objects around on the surface, but also to stack them (implying vertical movement). Thus, an interaction technique is needed that provides the full six degrees of freedom (three translation and three rotation axes). We use sticky fingers and opposable thumbs [14], which is an extension of the three-touch technique by Hancock et al. [12] and the proxy technique by Wilson et al. [31]. This technique uses the order of contacts (assigned per object to allow multi-object and multi-person interaction) to provide all degrees of freedom: the first touch provides translation in $2 \mathrm{D}$ (Figure $3 \mathrm{a}$ ), the second touch provides rotation about $z$ (Figure $3 \mathrm{~b}$ ) and movement along the $z$-axis (Figure 3c), and the third touch provides rotation about the $x$ - and $y$-axes (Figure 3d). The technique also uses 'crossing' (for each contact) to allow acquisition of small objects.

Many figurines, such as human figures, will often be used in a standing position. Because it is difficult or impossible to make figurines with a small base stand upright, we add invisible pedestals at the bottom of these objects. The pedestals are configured to collide only with the ground, and thus do not cause unexpected interactions with other figurines.

\section{Expressiveness Through Physics}

One of the key advantages of the described interaction technique is that it offers precise control over an object, further enabling the narrative (DC1). The use of a physics simulation, on the other hand, implies a certain imprecision and 


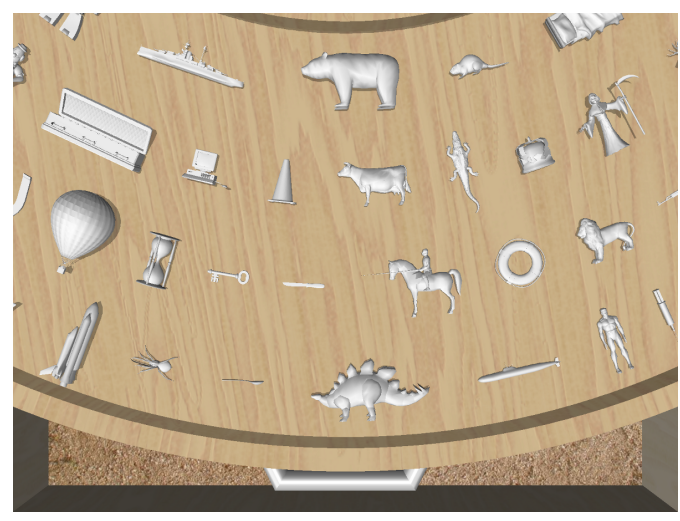

Figure 4: The ring-shaped figurine drawer.

lack of control. To get the best of both worlds, during interaction the object is put into a 'kinematic' state, essentially giving it infinite mass. Thus, the object is controlled only by the fingers and does not respond to forces in the physics engine, but all other objects are controlled by the physics engine and keep responding to the manipulated object. For example, it is possible to drag a figurine around, knocking over other figurines in its path, without losing control over the dragged object. This combination allows the therapist to interpret both the intended action on the object being controlled and the physical reaction of other objects. When an object is released, it retains the linear and angular velocity that it had in the previous animation frame. This allows objects to be tossed by moving them quickly, then releasing them. With some practice it is also possible to make an object spin or fly upwards, but this requires releasing two fingers within the same animation frame. Objects can be stopped by simply touching them. The 'crossing' feature also enables actions such as sweeping across the surface with the side of the hand. Although the actual interpretation of this gesture is very different from the proxy objects introduced by Wilson et al. [31], the net effect of objects being moved and pushing other objects ahead of them is similar.

By enabling these familiar physical interactions, we provide the client with a language for communicating to the therapist (perhaps subconsciously) through the virtual objects themselves (DC1). The therapist can then interpret what actions such as knocking over, tossing, and sweeping objects might mean about the client's psyche (DC4).

\section{Drawers and Tools}

To enable some of the digital extensions (DC3) and to support the accessibility of a wide range of figurines (DC2), we introduce drawers and tools. Each of these tools was designed to respond to the client's touches with the same interaction technique as the figurines, using the physics engine to impose constraints. We also designed the drawers to be able to slide in and out of view using a handle to save screen real estate. The drawers themselves can be tossed to quickly open and close them, and figurines will bounce around them in a natural way. Three such drawers are available: one for selecting figurines, one for resizing them, and one for painting on the sandtray floor.
Figurine Drawer. To allow the client to choose figurines for their story (DC2), the collection of figurines needs to be shown on the screen.

“... what really matters is that you're always looking at the toys themselves rather than some kind of menu system. So whether you go with scrolling, zooming or a 'drawer' metaphor, what is presented to the user should always be an image of some kind of toy or another. Otherwise I think you'd be inviting a really cognitive engagement with the system, which seems contrary to the unique genius of the thing to me." $\quad-\mathrm{T} 2$

Each figurine is shown as it would appear in the sandtray. However, the tabletop screen is not large enough to show all figurines at the same time with sufficient detail. We used a ring-shaped drawer similar to a 'Lazy Susan', only a small portion of which is visible at any given time (Figure 4). This allows an unlimited number of figurines to be accessed. When a figurine is removed from the drawer by dragging (using the sticky fingers and opposable thumbs technique), a copy will remain behind, allowing for quick and intuitive duplication. Figurines can be removed from the scene simply by putting them back into the drawer. This wide range of figurines is beneficial for therapist's observations, as the client has more choice about which figurines to pick. Therapists can also observe the client's browsing and decision-making processes, potentially involving inclusion, exclusion, and/or copying of figurines.

Resizing Drawer. To provide the client with the (physically impossible) ability to resize rigid objects (DC3), we provide a resize drawer. An alternative way to implement this is the two-finger 'pinch' gesture, in which two points on the object are pulled apart or pushed together to grow or shrink the object, similar to zooming. However, this gesture is already mapped to vertical movement of the figurine. The use of buttons or handles on the object would be harmful to the sense of physical realism and might be easy for the therapist to miss and therefore impede interpretation.

Instead, a drawer was added to the right side of the screen which acts as a 'resizing box'. One or more figurines can be put into the drawer. A dial is provided on the side of the drawer, with a ridged surface to suggest the ability to turn it. The dial can be turned using a single finger. When it is turned to the left, the figurines in the drawer shrink; when it is turned right, they grow (Figure 5). A minimum and maximum size are provided to prevent figures from shrinking out of sight or from becoming bigger than the display.

Painting Drawer. To provide the client with an open medium that can be shaped at will (DC2), we provide them with the ability to paint the background with different textures. On first thought, the interaction to 'paint' on a touch sensitive display could simply be like fingerpainting: wherever the surface is touched, the chosen paint colour appears. However, combining this technique with the other interactions in the virtual sandtray would require a mode switch, which would likely be problematic [27], and particularly when multiple people are using the system (DC4). 

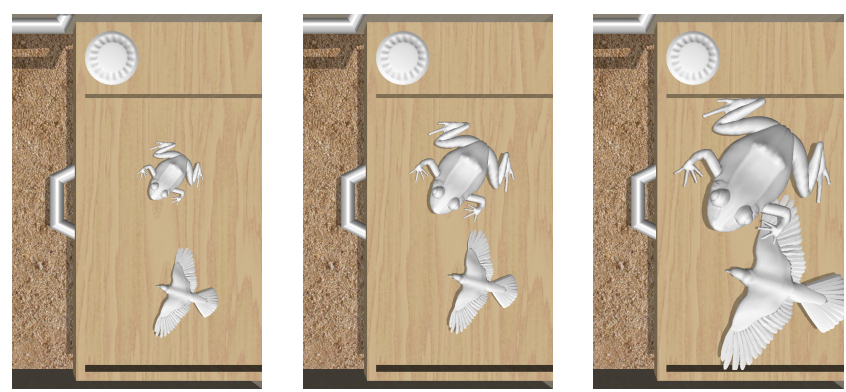

Figure 5: The resizing drawer in three different stages. Upon spinning the dial, the figurines inside the drawer will smoothly grow or shrink.

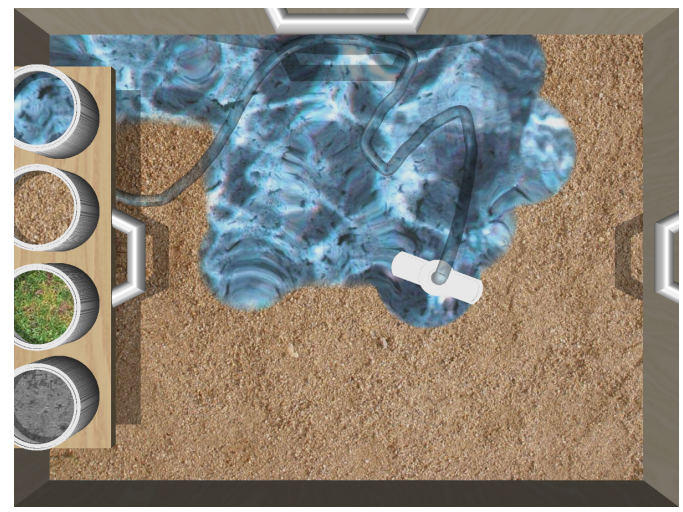

Figure 6: The painting system. In the drawer on the left, four buckets of paint can be seen. The hose connects the bottom of 'water paint' bucket to the nozzle, from which the water texture flows.

The painting ability is provided through the use of a spray nozzle tool. This nozzle can paint a texture on the floor (Figure 6). When the nozzle is picked up, it will rotate to point at the sandtray floor, which is the orientation in which it will normally be used. When the nozzle is lifted up, away from the floor, the region that is painted becomes larger.

The other part of the painting system is a drawer containing buckets of paint. A hose running from the drawer to the nozzle serves as a visual cue that they are related. A bucket can be selected by touching it. As long as the bucket is touched and the hose nozzle is in use, the texture paint it contains will flow through the hose and out the nozzle. Usually, the dominant hand is used to move the nozzle, while the non-dominant hand controls the paint selection. Paint will flow from the nozzle when both the nozzle and a paint bucket are being touched simultaneously. The nozzle can be moved around without painting by releasing the bucket.

Both the resize drawer and the painting drawer involve very explicit actions on the part of the client. The system's reaction to these actions (i. e., through the physics engine) makes the consequence of those actions available for interpretation by the therapist (DC4).

\section{PHASE III: FACE-TO-FACE FEEDBACK SESSION}

To validate and iterate on our design, we performed a daylong session with two sandtray therapists together with three of the designers. Neither therapist had any previous experience with digital tables. As previously stated, the focus of our research was to support the ability for therapists to recognize and interpret the actions of the client in a way that was valuable for understanding more about the child's psyche. We designed this face-to-face session to address the following questions:

- Can the therapists interpret the actions of a person using the virtual sandtray and use them to gain insight about their psyche?

- How can the design be improved to better enable the therapists to gain insight about a client's psyche?

Due to the fact that these therapists were typically distance collaborators, we also took this opportunity to share domain knowledge. While we had been iteratively discussing the prototype design remotely, this was the first face-to-face opportunity to do live demonstrations by both groups.

\section{Activities}

In this day-long session, the sandtray therapists participated in several activities: the therapists gave a demonstration of sandtray therapy to the designers using traditional physical figurines, the designers gave a demonstration of the virtual sandtray prototype, the therapists conducted a mock therapy session with the digital prototype, and all participated in a follow-up interview/brainstorming session.

Physical Demonstration. We began with a demonstration and instruction session from the perspective of the sandtray therapists. In this part, the sandtray therapists described how they performed sandtray therapy using physical figurines and instructed the designers about the theory, logistics, purpose, and essential components of the process of sandtray therapy. We asked questions whenever something was unclear and took written notes.

Prototype Demonstration. The designers provided an indepth demonstration of the digital sandtray prototype. We spent approximately one hour explaining the details of how to interact with our prototype and allowed both therapists to experience using the system. We discussed our design decisions, as well as several viable design alternatives. The therapists were encouraged to share their thoughts and comments. This part was videotaped.

Mock Therapy. Following the demonstration and a lunch break, with the warning from the therapists that "some therapy might happen", a designer played the role of the client in a mock therapy session with a therapist, as the other three participants (one therapist and two designers) observed. This session lasted about an hour and was also videotaped.

Interview and Brainstorming. For the remainder of the day (around 4 hours, including breaks), the two therapists and three designers participated in an interview and brainstorming session. The interview began with several planned questions, and continued with an unstructured discussion of the benefits, limitations, and next steps for future designs of digital sandtray therapy. Designers again took written notes and this part was not videotaped. 


\section{RESULTS \& DISCUSSION}

Of central import to the sandtray therapists, throughout all three phases, was the issue of understanding the client's psyche. An essential component of the exercise of sandtray therapy is to give the therapist insight into what the client is thinking and feeling through how they interact with objects. While sandtray therapists typically use physical props to gain this insight, our design revealed that this process is also possible with virtual ones. Instead of simply being a digital representation of some underlying data or model, the virtual objects in our system can take on symbolic meaning in the same way that physical ones do, to the level of providing access into the mind of someone interacting with them.

In this section, we first describe what our research revealed to be the essential components of interaction with virtual objects. These essential components allow virtual objects to cross the boundary from a digital representation to something that can allow the therapist access to the client's psyche. We then describe specific design refinements of our prototype that could address some of the issues that arose in our iterative process. Note that in this section we are discussing the results across all three phases of our design.

\section{Use of Virtual Objects}

There were several repeating themes about how a client's interaction with objects can help the therapist gain some insight about what they are thinking or feeling. These themes include construction, storytelling, actions, and arrangement.

Construction. The therapists frequently identified the ability to construct as an important aspect of the client's interaction with the sandtray. Construction is important because it "stimulates imagination", and in stimulating imagination, the therapist can better access the client's psyche. Several different forms of construction became apparent in our sessions.

The first and most obvious form of construction was the construction of the scene itself. The ability to freely move and rotate the figurines made it possible to create a scene that was composed of many different parts. The ability to make multiple copies of a specific figurine played a key role in this construction. For example, the client in our mock therapy session placed four palm tree objects of different sizes around an oasis. The therapist noted that the number and size of these palm trees matched the number of members in the client's family. Furthermore, the therapist suggested that this oasis may suggest that the family made the client feel safe. This example demonstrates that this form of construction, made possible by our prototype, was a sufficiently rich interaction for the therapist to gain insight.

Another key form of construction that was described by the therapists was the ability to create barriers. This form of construction was not directly supported by our prototype and implies that another level of granularity (besides that of the figurines) might be appropriate, where the virtual objects that the client can interact with can be bent, folded, or attached to one another, like a fence or bricks.

Storytelling. Another important aspect of the sandtray interaction was the ability for both the therapist and client to "tell a story". This storytelling process might be brought about in a variety of ways. For instance, the client might be encouraged to just play in the sandtray, and then the therapist might ask the client to explain the scene or elaborate on a specific object and discuss what it means to them.

A key aspect of this storytelling is that the objects in the scene can take on a variety of meanings. On the one hand, an airplane can represent just an airplane (i. e., itself), but on the other hand, it could represent a more abstract idea in the client's mind, such as flight or a desire to escape from something. The therapist's experience with the virtual sandtray prototype led them to state that they could easily interpret these different meanings from the virtual objects.

Actions. The therapists also discussed several ways in which the actions that the client uses to interact with the objects can be key to gaining insight about their psyche. The most commonly mentioned action from physical sandtray therapy was the idea of burying an object. This burying process can vary in meaning from client to client; it can imply things that the client wishes to hide or keep secret, or it can be a sign of aggression (e. g., suffocating). The ability for the therapist to observe this burying process is fundamental to the therapy session and was described as important to the therapist-client collaboration for which we are designing. Our current prototype does not allow for this burying interaction, as our choice of physics engine does not provide this functionality.

Other actions that may be relevant to the therapy were made available through the combination of our interaction technique with the physics engine. In particular, the ability to knock over one object with another, the ability to place an object inside another, and the ability to toss an object across the screen or drop an object from high above are actions that a client may do and can help the therapist to understand what is going on in the client's mind when they create a scene.

Arrangement. The arrangement of objects in the scene was also described as being highly important to the therapy process. In using our prototype, the therapists felt confident that clients would be able to easily and freely arrange objects in a way that would be useful for a therapy session. Although we did not perform a formal evaluation of usability, we interpret this confidence together with previous formal studies [12,31] as a sign that the interaction technique that we included in our prototype was sufficient for the type of object arrangements that the sandtray therapy requires. Furthermore, the use of gravity through the physics engine and pedestals on the base of each figurine facilitated this arrangement process.

\section{Design Refinements}

In addition to the high-level results regarding the efficacy of virtual objects as meaning-carrying artifacts, we gathered feedback about our current design that will help to inform future iterations. The therapists' feedback suggests that these improvements would facilitate interpreting a client's actions.

Some words used by the therapists to describe the virtual sandtray prototype were "relaxing and pleasurable", "attractive", and "appealing". Both therapists commented that, in 
its current form, the prototype might perhaps already be usable for therapy. On the other hand, comments were made about the lack of sensory feedback: touch, sound, and even smell. However, the application was described as being "still quite tactile". An interesting point raised was that the virtual sandtray does not so much invite storytelling, but rather the construction of a static scene. In that light, it might be more related to art therapy, for example the making of a collage.

Figurines. A class of objects that was missing were arbitrary objects that could be brought in by the therapist or the client and play a more metaphorical role. A small cardboard box could serve as a house, a stick could be used as a sword, or a pine cone could represent a baby, covered by a handkerchief to represent a blanket. The inability to bring such objects in could be found to be limiting if many therapy sessions are performed with the same, limited collection of figurines. It was suggested that a possibility should be added to draw or otherwise create one's own figurines, but this would be difficult to implement in an intuitive fashion, but might be possible through Teddy [15] or ShapeShop [25]. An alternative would be to use a device such as the Microsoft Surface ${ }^{3}$ capable of recognizing physical objects and have the physical props interact with the virtual ones in our prototype.

The presentation of figurines in the drawer was identified as potentially problematic; once a figurine was selected, the lack of structure made it difficult to find related figurines. Although the therapists who participated in the feedback session (T1 \& T3) normally present the figurines in their therapy in an organized way (in contrast to T2), they did comment that the lack of ordering in the virtual sandtray prototype "stimulates more random aspects of the psyche". This difference in approach suggests that we should design for some choice on the part of the therapist in how the figurines are presented. We could add a way to configure whether the objects are randomly sorted or organized in some fashion. Alternatively, a hybrid approach could start with a random presentation, but allow the client to easily find related figurines once a few have been selected.

Vertical Movement. Several problems were noted in relation to vertical movement of figurines. First, with the current top-down projection, it is not clear that the object is actually moving up or down, instead of simply changing size. This confusion was strongly reflected in the terminology used while discussing this action; even though people know that the object is actually moving up and down in the scene, they often still talk about "making it bigger" and "making it smaller". This suggests a disconnect between the client's actions and the therapist's interpretation. This might partly be blamed on the fact that the shadow is cast directly below each object, which often causes the shadow to be partly or completely obscured. A second shadow, cast from the side, might improve interpretation of movement in $z$; a projection that is not strictly top-down could also help.

A second problem is that the 'sticky fingers' paradigm [14] implicitly makes lifting an object very sensitive. Especially

\footnotetext{
${ }^{3}$ Microsoft Surface. http://www.microsoft.com/surface
}

when the two fingers start close together, a small movement of the fingers will result in a large vertical motion. Doubling the distance between the fingers will move the object twice as close to the virtual camera, which is quite a large distance. Perhaps it is better to let go of the stickiness of one of the fingers. A more formal user study may be necessary to objectively determine which is better.

A third problem is that it is possible to move a figurine so that it becomes invisible. For example, a figurine can be pushed down right through a drawer from above, causing the figurine to become hidden underneath the drawer. While this 'feature' may be one way of achieving the burying action requested by the therapists, it may also be an unintended consequence of the client's actions, introducing ambiguity in how the therapist interprets the action. It might be better to keep a figurine always visible, by forcing it to be always above everything else or by using digital effects such as outlines, shadows or transparency.

\section{BEYOND THE DIGITAL SANDTRAY}

On the one hand, our digital sandtray prototype is a single point in the design space of interactive tabletops. Thus, our iterative process may not yield results that generalize beyond this design. However, our method is an example of how we would recommend designing future interactive tabletop systems and we would argue that many of the design decisions that we made can be adopted on other tabletop systems. Furthermore, our feedback session provides some of the first available evidence that virtual objects can be used in a real application in a very different way than they are typically used on desktop computers. In particular, the therapists in our study pointed out the following aspects of our system as particularly compelling:

Communication through virtual objects. The therapists stated that the 3D interaction (sticky fingers and opposable thumbs [14]) would be sufficiently rich for therapeutic purposes. Specifically, the therapists felt they would be able to gain insight about a client's psyche based on his or her interactions with a virtual object.

Deployable system. Our system is an example of an application that has been identified as usable as-is by the therapists. Our method of designing and developing this application in collaboration with sandtray therapists could be adopted for tabletop systems in other domains. Specifically, our work serves as a case study that cooperative design [9] may lead to successful interactive tabletop systems.

Repurposeable virtual objects. The therapists felt that the interaction with many individual 3D objects on an interactive table was both meaningful and usable. The therapists illustrated this during mock therapy showing how sometimes virtual objects were recognized as themselves (e.g., a rock is a rock), as well as metaphors, symbols, or archetypes (i.e., representations of things from a person's mind-for instance a rock could represent a member of one's family). This indicated that, in our system, virtual objects could be interpreted by an observer as more than just digital representations of data or information. 


\section{CONCLUSION}

In this paper, we have presented the viability of a virtual sandtray that has been identified as usable for therapy by domain experts. Beyond the specific domain of art therapy, this work also serves to inform the design process for tabletop display systems and provides some insight into how interaction with $3 \mathrm{D}$ objects on a table can be made useful in practice. Specifically, the use of precise interaction and a physics engine can together provide a richness that is sufficient for therapists to understand things about a client's psyche through their interactions with the virtual artifacts. These artifacts thus can take on meaning in a way that is not typically sought after in the design of traditional computer applications. Future designers of tabletop systems can use this work to inform how they can achieve similar levels of rich interaction, and therefore make a new type of interface between humans and virtual objects possible.

\section{ACKNOWLEDGEMENTS}

We would like to thank Natural Science and Engineering Research Council of Canada, Albertas Informatics Circle of Research Excellence, Alberta Ingenuity, and the Canadian Foundation of Innovation for research support. We also thank the reviewers and members of the iLab for their helpful comments on this work.

\section{REFERENCES}

1. Agarawala, A., Balakrishnan, R. Keepin' it real: pushing the desktop metaphor with physics, piles and the pen. In Proc. CHI, ACM (2006), 1283-1292.

2. Bers, M. U., Ackermann, E., Cassell, J., Donegan, B., Gonzalez-Heydrich, J., DeMaso, D. R., Strohecker, C., Lualdi, S., Bromley, D., Karlin, J. Interactive storytelling environments: coping with cardiac illness at Boston's Children's Hospital. In Proc. CHI, ACM (1998), 603-610.

3. Bradway, K., McCoard, B. Sandplay: Silent Workshop of the Psyche. Routledge, 1997.

4. Cao, X., Wilson, A. D., Balakrishnan, R., Hinckley, K., Hudson, S. E. ShapeTouch: leveraging contact shape on interactive surfaces. In Proc. Tabletop, IEEE (2008), 129-136.

5. Carpendale, M. Essence and Praxis in the Art Therapy Studio. Trafford Publishing, 2009.

6. Cassell, J., Ryokai, K. Making space for voice: technologies to support children's fantasy and storytelling. PUC 5, 3 (2001), 169-190.

7. Cunningham, J. (Ed.). Journal of Sandplay Therapy, volume 18, number 2. Sandplay Therapists of America, 2009.

8. Dietz, P., Leigh, D. DiamondTouch: a multi-user touch technology. In Proc. UIST, ACM (2001), 219-226.

9. Greenbaum, J. M., Kyng, M. (Eds.). Design at Work: Cooperative Design of Computer Systems. L. Erlbaum Associates Inc., 1991.

10. Grubert, J., Carpendale, S., Isenberg, T. Interactive stroke-based NPR using hand postures on large displays. In Eurographics Short Papers, Eurographics Association (2008), 279-282.

11. Han, J. Y. Low-cost multi-touch sensing through frustrated total internal reflection. In Proc. UIST, ACM (2005), 115-118.
12. Hancock, M., Carpendale, S., Cockburn, A. Shallow-depth 3D interaction: Design and evaluation of one-, two- and three-touch techniques. In Proc. CHI, ACM (2007), $1147-1156$.

13. Hancock, M., Carpendale, S., Vernier, F. D., Wigdor, D., Shen, C. Rotation and translation mechanisms for tabletop interaction. In Proc. Tabletop, IEEE (2006), 79-88.

14. Hancock, M., ten Cate, T., Carpendale, S. Sticky tools: Full 6DOF force-based interaction for multi-touch tables. In Proc. ITS, ACM (2009), 145-152.

15. Igarashi, T., Matsuoka, S., Tanaka, H. Teddy: a sketching interface for 3D freeform design. In Proc. SIGGRAPH, ACM/Addison-Wesley (1999), 409-416.

16. Kalff, D. M. Sandplay: A Psychotherapeutic Approach to the Psyche. Sigo Press, 1980.

17. Kruger, R., Carpendale, S., Scott, S. D., Greenberg, S. Roles of orientation in tabletop collaboration: comprehension, coordination and communication. J. CSCW 13, 5-6 (2004), 501-537.

18. Kruger, R., Carpendale, S., Scott, S. D., Tang, A. Fluid integration of rotation and translation. In Proc. CHI, ACM (2005), 601-610.

19. Krüger, W., Fröhlich, B. The Responsive Workbench. $C G \& A$ 14, 3 (1994), 12-15.

20. Li, Y., Fontijn, W., Markopoulos, P. A tangible tabletop game supporting therapy of children with cerebral palsy. In Proc. Fun and Games, Springer-Verlag (2008), 182-193.

21. Liu, J., Pinelle, D., Sallam, S., Subramanian, S., Gutwin, C. TNT: improved rotation and translation on digital tables. In Proc. GI, Canadian Information Processing Society (2006), 25-32.

22. Morris, M. R., Cassanego, A., Paepcke, A., Winograd, T., Piper, A. M., Huang, A. Mediating group dynamics through tabletop interface design. CG\&A 26, 5 (2006), 65-73.

23. Piper, A. M., Hollan, J. D. Supporting medical conversations between deaf and hearing individuals with tabletop displays. In Proc. CSCW, ACM (2008), 147-156.

24. Ryokai, K., Cassell, J. StoryMat: A play space for collaborative storytelling. In Proc. CHI, ACM (1999), 272-273.

25. Schmidt, R., Wyvill, B., Sousa, M. C., Jorge, J. A. ShapeShop: Sketch-based solid modeling with BlobTrees. In 2nd Eurographics Workshop on Sketch-Based Interfaces and Modeling (2005), 53-62.

26. Schuler, D., Namioka, A. (Eds.). Participatory Design: Principles and Practices. L. Erlbaum Associates Inc., 1993.

27. Sellen, A. J., Kurtenbach, G. P., Buxton, W. A. S. The prevention of mode errors through sensory feedback. HCI 7, 2 (1992), 141-164.

28. Tse, E., Shen, C., Greenberg, S., Forlines, C. How pairs interact over a multimodal digital table. In Proc. CHI, ACM (2007), 215-218.

29. Walter, K. http://en.wikipedia.org/wiki/File:Sandspiel1.jpg.

30. Walter, $\mathrm{K}$. http://en.wikipedia.org/wiki/File:Sandspiel_Figuren2.jpg.

31. Wilson, A. D., Izadi, S., Hilliges, O., Garcia-Mendoza, A., Kirk, D. Bringing physics to the surface. In Proc. UIST, ACM (2008), 67-76.

32. Wu, M., Balakrishnan, R. Multi-finger and whole hand gestural interaction techniques for multi-user tabletop displays. In Proc. UIST, ACM (2003), 193-202. 